\title{
Development of an immunofluorescent AR-V7 circulating tumor cell assay - A blood-based test for men with metastatic prostate cancer
}

\author{
David Lu ${ }^{1,2}$, Rachel Krupa ${ }^{1}$, Melissa Harvey ${ }^{1}$, Ryon P. Graf ${ }^{1}$, Nicole Schreiber ${ }^{3,4}$, Ethan Barnett ${ }^{3}$, Emily Carbone ${ }^{3}$, Adam Jendrisak ${ }^{1}$, \\ Audrey Gill' ${ }^{1}$, Sarah Orr ${ }^{1}$, Howard I. Scher ${ }^{5,6}$, Joseph D. Schonhoft ${ }^{1}$ \\ ${ }^{1}$ Epic Sciences, San Diego, California - USA \\ ${ }^{2}$ Exact Sciences, Madison, Wisconsin - USA \\ ${ }^{3}$ Department of Medicine, Memorial Sloan Kettering Cancer Center, New York, New York - USA \\ ${ }^{4}$ Prostate Cancer Clinical Trials Consortium, New York, New York - USA \\ ${ }^{5}$ Genitourinary Oncology Service, Department of Medicine, Memorial Sloan Kettering Cancer Center, New York, New York - USA \\ ${ }^{6}$ Department of Medicine, Weill Cornell Medical College, New York, New York - USA
}

\begin{abstract}
Introduction: Here we describe the development of a protein immunofluorescent assay for the detection of nuclear-localized androgen receptor variant 7 (AR-V7) protein within circulating tumor cells (CTCs) identified in patient blood samples. Used in the clinic, the test result serves as a validated biomarker of futility for patients with progressing metastatic castration-resistant prostate cancer (MCRPC) who are treated with androgen receptor targeted therapies (AATT) in whom nuclear-localized AR-V7 CTCs are identified and have received level 2A evidence in the 2019 National Cancer Center Network (NCCN) guidelines (v1.0).

Methods: Assay development was completed on the Epic Sciences rare cell detection platform using control cell lines of known AR-V7 status and clinical testing of mCRPC patient samples obtained at the decision point in management.

Results and conclusions: Using these samples, all assay parameters, scoring criteria, and clinical cutoffs for positivity were prospectively selected and locked. After assay lock, blinded clinical validation testing was initiated on multiple, independent, clinical cohorts as reported by Scher et al (JAMA Oncol. 2016;2:1441-1449; JAMA Oncol. 2018;4:1179-1186) and Armstrong et al (J Clin Oncol. 2019;37:1120-1129).
\end{abstract}

Keywords: AR-V7, Castration-resistant prostate cancer, Circulating tumor cells (CTCs), Predictive biomarkers

\section{Introduction}

The last decade has seen a dramatic expansion of the therapeutic options for men with recurrent metastatic castration-resistant prostate cancers (mCRPCs). In particular, next-generation androgen receptor targeted therapies (AATT) including abiraterone, enzalutamide, apalutamide, and darolutamide have unequivocally demonstrated that many CRPC

Received: June 9, 2020

Accepted: September 4, 2020

Published online: October 23, 2020

Corresponding author:

Joseph D. Schonhoft, PhD

Epic Sciences

9381 Judicial Dr.

San Diego, California 92121, USA

joseph.schonhoft@epicsciences.com cancers remain dependent on androgen receptor signaling for growth and that these orally administered agents can improve progression-free survival and overall survival (1-7).

AATTs have quickly become the most common treatment modalities in CRPC. Abiraterone and enzalutamide received approvals in 2011 and 2012, apalutamide in 2019, and darolutamide the same year (1-7). Since then, a recent analysis of medical records of 2,559 mCRPC patients in the United States from January 2013 to September 2017 found that 1,980 (77\%) received life-prolonging therapies, of whom 1,294 (65\%) received an AATT as the first-line therapy. Of the 969 patients who received a second-line treatment, $523(54 \%)$ received an AATT, with sequential use of AATTs commonly practiced (8). The second most common class of drugs utilized are taxanes, such as docetaxel or cabazitaxel (8).

While these two drug classes are the most commonly utilized in the management of MCRPC in the United States, the choice between an AATT and a taxane is often empiric without a predictive biomarker guidance (8). In this setting, the most clinically validated treatment selection biomarker 
to date is the presence of nuclear-localized androgen receptor splice variant 7 (AR-V7) protein detected in the circulating tumor cells (CTCS) of metastatic CRPC patients using the Epic Sciences platform, receiving level 2A evidence in the 2019 National Cancer Center Network (NCCN) guidelines. The assay is performed at Epic Sciences as a laboratory developed test (LDT), reimbursed by the Centers for Medicare and Medicaid Services (CMS) and top private payers, has received accreditation by the College of American Pathologists (CAP), and approval from the New York State Department of Health (NYSDOH).

The first study to demonstrate clinical utility of the Epic Sciences nuclear-localized AR-V7 test was a blinded crosssectional study consisting of mCRPC patients treated with AATT and taxanes from Memorial Sloan Kettering Cancer Center (MSKCC) (9). Both treatment-naïve (first line) and pretreated (second line or greater) patients were considered, and the results showed superior survival times for patients in whom nuclear-localized AR-V7 CTCS were identified who received a taxane compared with AATT and that favorable prostatespecific antigen (PSA) responses were observed only in the patients treated with taxanes. This study was followed by a blinded prospective validation that included $\mathrm{MCRPC}$ patient samples from three centers (10). Here, only second-line mCRPC-treated patients, those who had failed one prior therapy, were considered and AR-V7 biomarker-positive patients were observed to have favorable survival on taxanes compared with AATT in a risk-adjusted model, confirming our initial report. The third study, PROPHECY (NCT02269982) (11), was a prospectively designed multicenter blinded validation that included first- and second-line MCRPC patients with a poor prognosis based on a validated nomogram that included pretreatment levels of lactate dehydrogenase (LDH), alkaline phosphatase (ALK), or presence of visceral metastases among other features. All patients were treated with AATT. Here again, patients with nuclear-localized AR-V7 CTCs had a shorter time to radiographic progression, shorter overall survival, and an unfavorable PSA response (11).

Here we present the development data for the Epic Sciences nuclear-localized AR-V7 assay. Analytical and clinical feasibility testing, including control cell line screening, antibody testing, and initial patient feasibility testing, were executed to achieve assay lock prior to the commencement of the three clinical utility studies.

\section{Materials and methods}

\section{AR-V7-positive cell line engineering}

A lentiviral vector containing both EF1a-AR-V7 and RSVpuromycin inserts was transduced into a PC3 (AR-negative) parental cell line to create a stable AR-V7 expressing cell line. Target sequence integration into the cell line genome was confirmed using genomic template polymerase chain reaction (PCR). AR-V7 transfected cells were maintained under puromycin selection during culture to ensure the stable expression of AR-V7 protein. Transfected subclones were assessed via quantitative PCR (qPCR), Western blotting and immunofluorescent staining to confirm AR-V7 protein expression.

\section{Cell culture}

All cell lines were cultured using sterile techniques and complete cell culture media. To manufacture control slides for assay development and subsequent assay validation, cells were trypsinized into single-cell suspension, counted, and spiked into normal donor (ND) blood collected into Streck $\mathrm{BCT}$ at known quantities.

\section{$q P C R$}

qPCR primer pairs specific for the AR N-terminal domain (ARNTD), AR-V7 cryptic exon (ARV7CE), and the AR C-terminal domain (ARCTD) were used to assess appropriate gene expression and messenger ribonucleic acid (mRNA) splicing profiles in each cell line.

Cells were harvested and assayed for gene expression using a Single Cell-to-CT Kit (Ambion) as per manufacturer protocol. Real-time quantitative (qRT)-PCR was performed with TaqMan probes for ARNTD, ARCTD (ThermoFisher), or a probe set specific to ARV7CE (custom design) with a QuantStudio 7 (ThermoFisher). All samples were analyzed for $18 \mathrm{~S}$ (ThermoFisher) as an internal RNA quality control.

\section{Western blotting}

Whole cell lysates were prepared using NuPAGE LDS sample buffer supplemented with $0.5 \%$ beta-mercaptoethanol and protease inhibitor. Cells were harvested via scraping from tissue culture dishes at approximately $80 \%$ confluency and sonicated via probe sonicator followed by incubation at $95^{\circ} \mathrm{C}$ for 5 minutes.

Protein lysates (40 $\mathrm{\mu g} /$ well) were run on NuPAGE $4 \%-12 \%$ Tris-Glycine precast gels and transferred using the iBlot Dry Blotting system. Overnight incubation with AR-V7 primary antibody followed by detection via WesternDot 625 goatantirabbit kit (Cat\# W10142, ThermoFisher) was used for visualization of $A R-V 7$ protein.

All Western blotting reagents and equipment was sourced from ThermoFisher and AR-V7 antibody was sourced from Abcam (clone EP343).

\section{Laboratory-derived sample and patient blood sample processing}

Laboratory-derived (LD) control samples were created using ND blood collected into Streck cell-free blood collection tubes and spiked with either positive (GS3 or 22RV1) or negative (DU145 or PC3) cell line cells (CLCS). Contrived LD samples were used to create unequivocal positive and negative samples to mimic patient samples of known AR-V7 status. These samples were accessioned and processed identically to patient samples. For patient samples, mCRPC patient blood $(7.5 \mathrm{~mL})$ was collected in Streck tubes at MSKCC and processed at Epic Sciences within 48 hours. All patients gave informed consent to an institutional review board (IRB)-approved protocol before blood draw. For both LD and patient samples, red blood cells were lysed, and approximately 3 million nucleated cells were dispensed onto glass microscope slides, 
fixed, and stored at $-80^{\circ} \mathrm{C}$ as previously described (9-13). All testing presented in this study was performed prior to the start of the three clinical validation studies.

\section{ND blood sample processing}

ND blood samples were acquired from a local blood collection site (The Scripps Research Institute Normal Blood Donor Service, La Jolla, CA). Blood was drawn into Streck tubes from male donors and shipped to Epic Sciences for processing.

\section{Immunofluorescent staining}

Briefly, the immunofluorescence (IF) staining components of the assay are as follows and as previously reported (9-13). Analytical validation of the Epic Sciences platform for rare cell detection has been previously reported (12). All nucleated cells deposited onto a slide are detected with DAPI, a dye that fluoresces upon binding to deoxyribo nucleic acid (DNA) and stains the cell nucleus. White blood cells (WBCs) are detected using an anti-CD45 antibody that is directly conjugated to Alexa Fluor ${ }^{\text {TM }}$ 647. CK proteins in CTCs are detected using a panel of mouse immunoglobulin G (IgG)1 anti-CK antibodies visualized with a secondary goat antimouse IgG1 antibody conjugated to Alexa Fluor ${ }^{\mathrm{TM}}$ 555. The rabbit monoclonal anti-AR-V7 antibody is recognized by a secondary goat antirabbit antibody conjugated to horseradish peroxidase (GARHRP), which catalyzes covalent binding of tyramide conjugated to Alexa Fluor ${ }^{\mathrm{TM}} 488$.

\section{Immunofluorescent scanning}

Stained slides are scanned using the Epic Sciences automated scanning platform. The platform consists of a highthroughput microscope slide scanning system that collects images at 10x magnification for each of the four fluorescent channels used in the AR-V7 assay (DAPI, CK, CD45, AR-V7). All nucleated cells found on each glass slide are imaged and mean fluorescent intensities (MFIs) are quantified. Final outputs from the fluorescent scanner include cell-level images enabling visualization of subcellular biomarker localization, MFIs for each biomarker, and unique coordinates to allow for the relocation of cells of interest for genomics or reimaging purposes.

\section{Imaging algorithms}

Using the images captured by the Epic Sciences platform, proprietary image analysis algorithms classify CTCs based on cellular morphology and biomarker MFIs detected from each of the four channels corresponding to DAPI, CK, CD45, and AR-V7. Exposure times are automatically varied to maximize signal in each field of view. For this reason, the signal quantified in a CLC or CTC subpopulation is normalized to the average signal observed in a representative population of WBCs on the slide.

The nuclear localization of AR-V7 signal (NL-ARV7) is determined based on its colocalization with DAPI (13). On a
CTC level, results are binary for the purposes of this assay. A CTC with AR-V7 fluorescent signal above background that colocalizes with DAPI is considered AR-V7 positive. Trained technical reviewers confirm the algorithm classification to identify the presence or absence of AR-V7-positive (nuclearlocalized) CTCs.

\section{Results \\ Control cell line characterization of AR-V7 mRNA and protein expression}

To enable AR-V7 IF assay development, appropriate control cell lines (PC3, DU145, and 22RV1) were screened to confirm AR-V7 mRNA and protein expression levels. Consistent with previous reports, PC3 and DU145 cells lacked detectable AR gene expression and were negative for both AR mRNA and protein (Fig. 1A, B). 22RV1 cells are known to express several AR splice variants (14), and because of this heterogeneity, mRNA transcripts corresponding to the ARNTD-, ARV7CE-, and ARCTD-specific sequences were detected, reconfirming the expression of both full-length AR and AR-V7 splice variants (Fig. 1A).

To create an unequivocal control without the limitations of the inherent heterogeneity and lot-to-lot variability of AR variant expression in 22RV1s, we engineered a cell line to constitutively express only the AR-V7 variant. Lentiviral transfection of a PC3 parental line was used to generate several candidate subclones for constitutive AR-V7-expressing cell lines in which three were selected (GS1, GS3, GS8). While all three subclones expressed the AR-V7 transcript, only the GS3 line was found to be positive for $A R-V 7$ protein via IF staining (Fig. 2A) and Western blot (Fig. 1B). Notably, full-length $A R$ is not expressed in GS3 cells as evidenced by lack of detectable ARCTD transcript (Fig. 1A). For subsequent AR-V7 IF assay development, both PC3 and DU145 cells were utilized as negative controls to confirm test specificity, while 22RV1 and GS3 cells were used as positive controls.

\section{AR-V7-specific immunofluorescent assay development}

AR-V7 IF assay development utilized the Epic Sciences existing rare cell detection platform as previously described (12). Using LD samples containing either positive (GS3 or 22RV1) or negative (PC3, DU145) cells spiked into ND blood, or ND blood alone, IF staining conditions were developed and subsequently optimized (Fig. 2). Primary antibody titration results observing relative fluorescent signal in each CLC as a function of primary antibody concentration are shown in Figure 2B, C.

Upon completion of preliminary primary and secondary antibody titration curves, extensive guard banding studies were performed to confirm optimal assay parameters including primary and secondary antibody concentrations, tyramide concentration, fixation reagent concentration, fixation reagent incubation time, and wash buffer incubation times (data not shown). Final assay conditions were selected based on optimal signal-to-background ratios, yielding median 
A
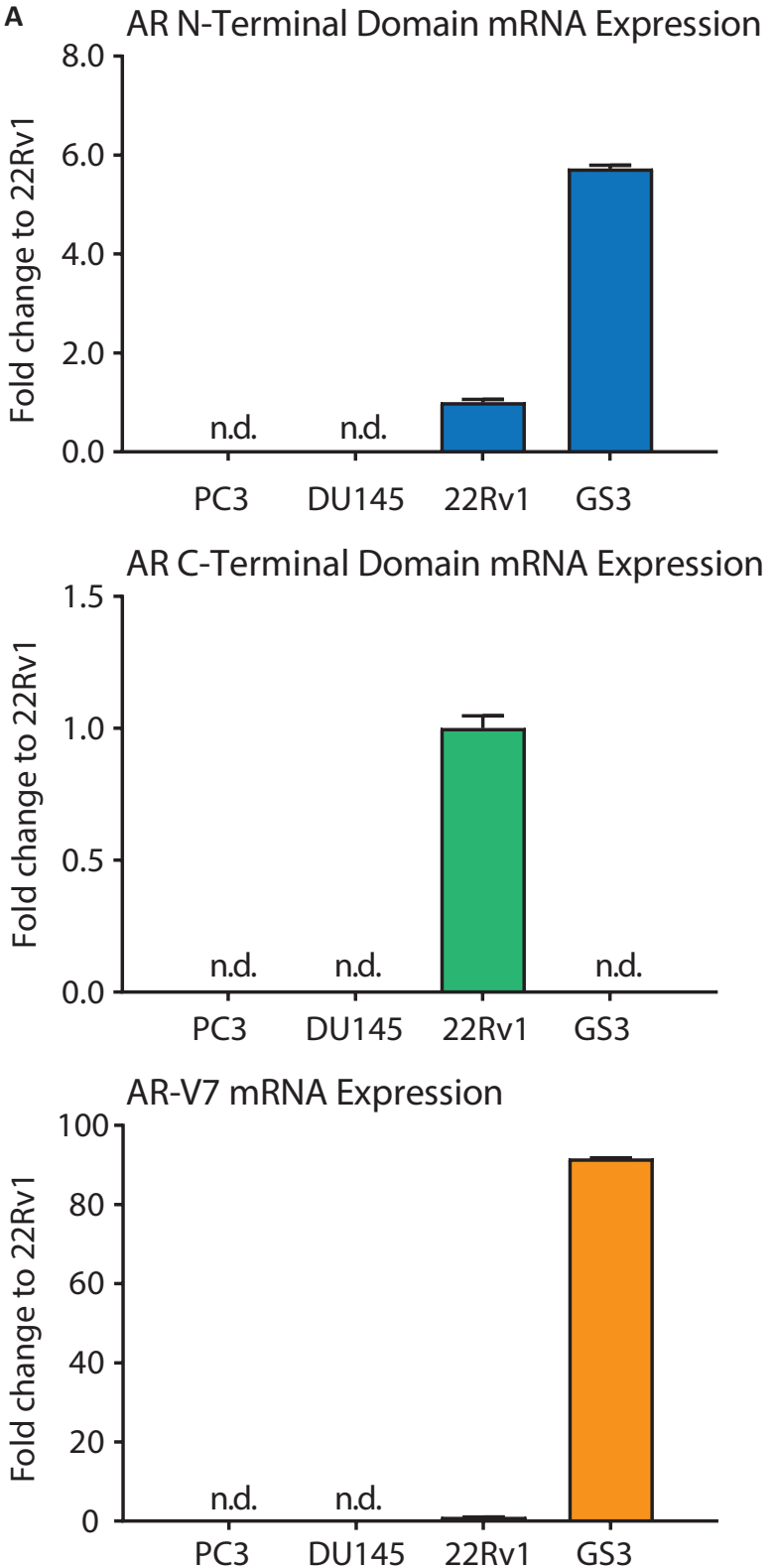

B

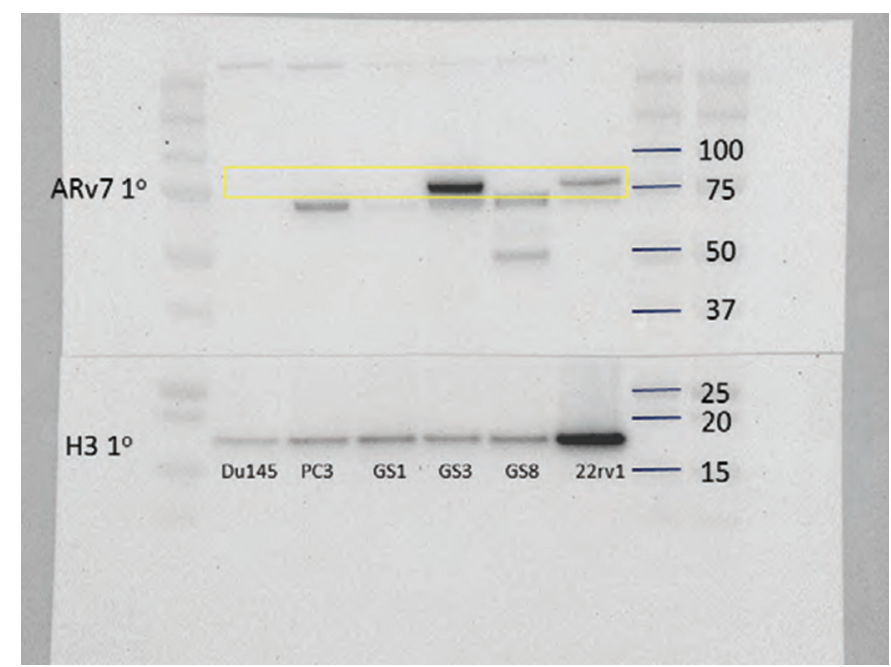

Fig. 1 - Control cell line characterization of androgen receptor variant 7 (AR-V7) messenger ribonucleic acid (mRNA) and protein expression. (A) Quantitative polymerase chain reaction analysis using AR N-terminal (ARNTD), AR C-terminal (ARCTD), and AR-V7 (ARV7CE)specific primer sets. PC3 and DU145 cell lines are negative for all AR-specific mRNA sequences (ND: not detected). While 22RV1s expresses relatively low levels of AR-V7 transcript, 90 -fold relative abundancy was observed in AR-V7 stably transfected GS3 cells. Furthermore, no ARCTD-specific transcript was observed in GS3s, confirming no full-length AR mRNA expression. mRNA abundance is quantified as fold expression compared to 22RV1. 18S is used as an internal control. (B) Western blot confirms mRNA expression profiles. DU145 and PC3 cells are AR-V7-negative, while abundant protein was observed in GS3 and 22RV1 cell lysates. GS1 and GS8 cells, alternative AR-V7 stably transfected subclones, did not produce AR-V7 protein and were discarded. Lower weight nonspecific bands ( 72 kDa, $50 \mathrm{kDa})$ were observed in some cell lines. Histone $\mathrm{H} 3$ is used as a loading control.

AR-V7 fluorescent signals of 8.4-, 17,- and 127-fold above background in DU145, 22RV1, and GS3 cells, respectively (Fig. 2C). AR-V7 signal in positive control cells was observed to be consistently and predominantly localized in the cell nucleus, whereas signal in DU145 and PC3 was largely undetectable and if low levels were present it was non-nuclear (example images, Fig. 2A). Therefore, hereafter, we require nuclear-specific localization of AR-V7 to call a cell positive. By comparison, similar criteria for nuclear localization have been applied to AR-V7 expression in tissue (15-17). Finally, we tested 21 ND blood donors and 0/21 (0\%) were found to have AR-V7-positive CTCs. 
A

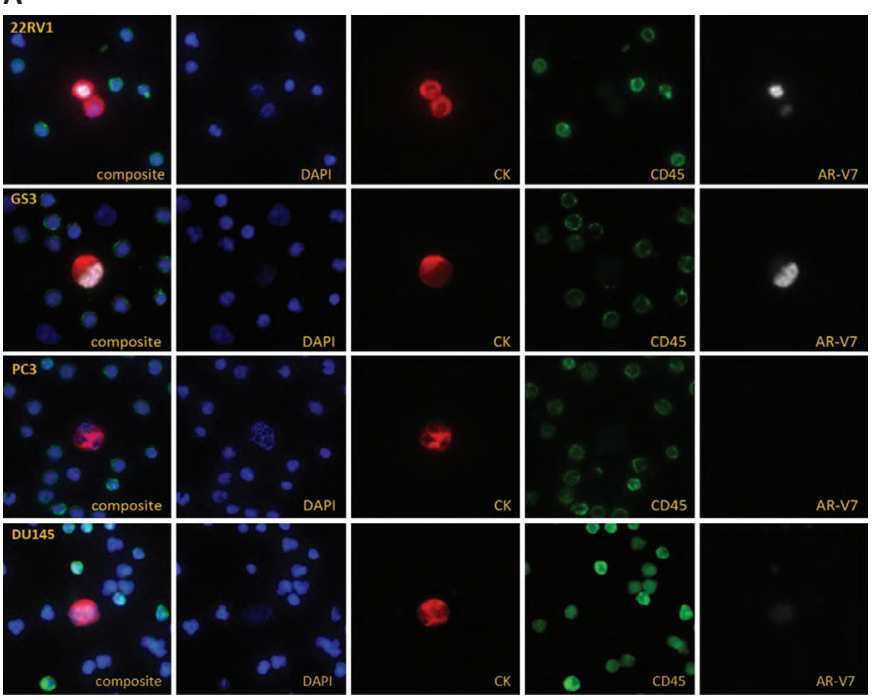

B

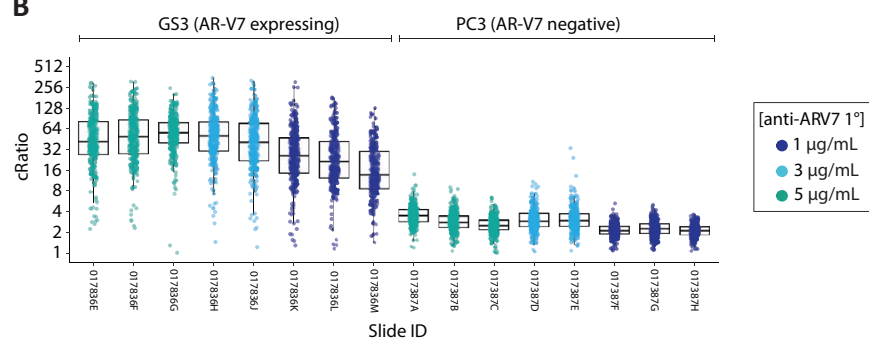

C

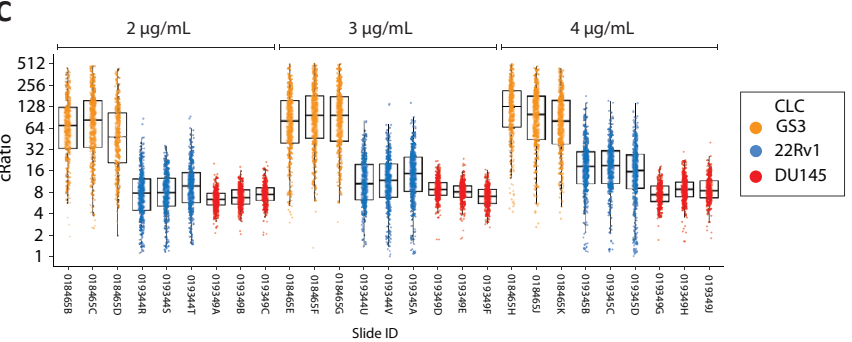

Fig. 2 - Androgen receptor variant 7 (AR-V7)-specific immunofluorescent assay development. Optimization of an immunofluorescent circulating tumor cell (CTC) assay detecting the presence of nuclear-localized AR-V7 protein was developed using control cell lines. Nuclearlocalized staining was observed in 22RV1 and GS3 cells, whereas PC3 and DU145 cells are consistently negative for nuclear AR-V7 signal as seen in representative images (A). Representative microscopy images of 22Rv1, GS3, PC3, DU145 cell lines. (B) Antibody titration curves comparing GS3 (AR-V7 expressing) and PC3 (AR-V7 negative). Each data point represents the mean fluorescence signal relative to the local background of a single cell detected (cRatio). (C) Titration curves comparing GS3, 22RV1, and DU145 at 2, 3, and $4 \mu \mathrm{g} / \mathrm{mL}$ of AR-V7 primary antibody. At the selected primary antibody concentration of $3 \mu \mathrm{g} / \mathrm{mL}$, typical fluorescent signals observed in DU145, 22RV1, and GS3 were 8.4-, 17-, and 130-fold above background, respectively. Immunofluorescent signals in negative controls (i.e., PC3, DU145) are nonzero due to a combination of cellular autofluorescence, instrument noise, and nominal nonspecific (non-nuclear) antibody binding.

\section{Scoring criteria for AR-V7-positive cells}

Before initiation of patient feasibility testing, scoring criteria were defined. For a CTC to be scored as AR-V7 positive, it must: (1) be negative for CD45 staining (blood lineage marker); (2) have CK positivity or cellular morphology indicative of epithelial (or nonhematopoietic) lineage; (3) have an intact nucleus without signs of apoptosis; and (4) have AR-V7 staining with clearly defined nuclear-localized signal. Existing clinical data indicated that AR-V7 would be more likely to be expressed in patients who had previously failed treatments in the metastatic setting (18).

To assess the general clinical feasibility of the AR-V7 assay to potentially detect physiologically relevant levels of AR-V7 protein in CTCs, 27 mCRPC patient samples were tested. These samples were obtained from patients who had previously failed at least one line of treatment in the metastatic setting, were progressing, and in need of a therapy change. Samples were not selected based on known AR-V7 status. Furthermore, this cohort was used strictly for development purposes and was not included in any subsequent clinical validation testing. Twenty-two of 27 (82\%) patients harbored CTCs, 14/27 (51.8\%) of patients harbored CTCs with nuclearlocalized expression, and patients with AR-V7-positivity qualitatively demonstrated substantial heterogeneity, in terms of AR-V7 protein expression localization and intensity within their observed CTC populations (Fig. 3).

Based on these data, a cutoff of at least 1 AR-V7-positive CTC detected in a patient sample was prospectively chosen as the criterion for AR-V7 test positivity. For each patient sample here, 6 million total nucleated cells were analyzed per patient sample. The detection of at least 1 positive cell was used for all subsequent clinical validation studies (9-11). Furthermore, a nuclear AR-V7 fluorescence intensity of at least 3.2-fold above background was also prospectively selected as a criterion for AR-V7 positivity based on a qualitative assessment of the MCRPC patient CTCs, that is, it was not possible to determine AR-V7 localization below this threshold. Therefore, patient samples containing CTCs with AR-V7 IF intensity below 3.2, those with non-nuclear-localized signal above 3.2, and those samples in which CTCs are not detected are classified as negative.

\section{Discussion}

In this report we present assay development results for the Epic Sciences nuclear-localized AR-V7 test. Importantly, all studies presented and all assay parameters were locked prior to initiation of any of the three clinical utility studies (9-11). The assay specifically detects the AR-V7 splice variant protein lacking the ligand binding domain in the nucleus of CTCs. Control cell line experiments demonstrate the assay's ability to distinguish between the AR-V7 truncated protein from the full-length protein and other splice variants within CTCs. Analysis of blood samples from patients with progressing $\mathrm{MCRPC}$ in need of a change in therapy for progressing disease were used to develop a patient scoring criteria and to demonstrate the ability of 
A

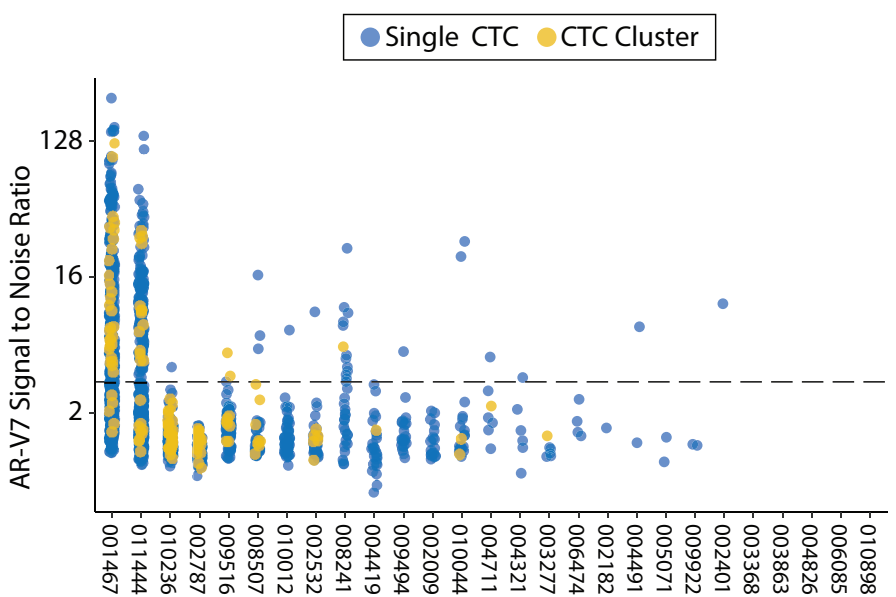

Inidividual Blood Tube ID From a mCRPC Patient
B

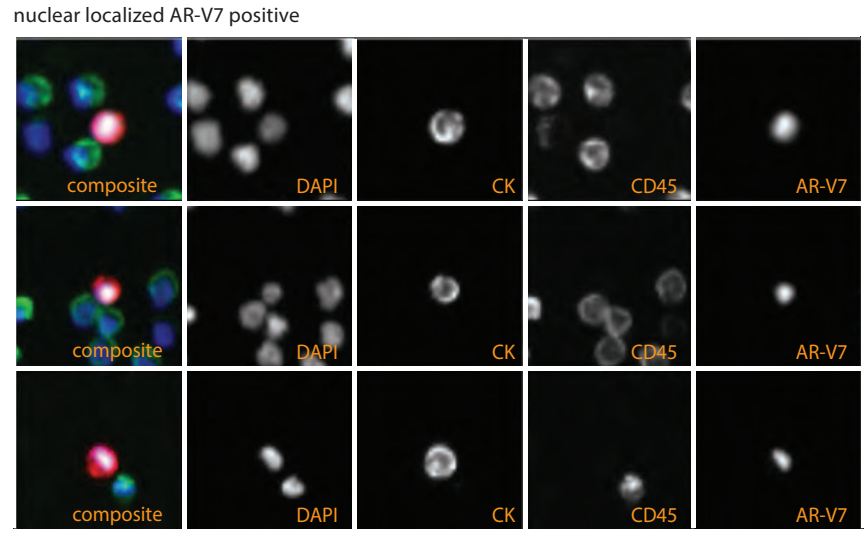

nuclear localized AR-V7 negative

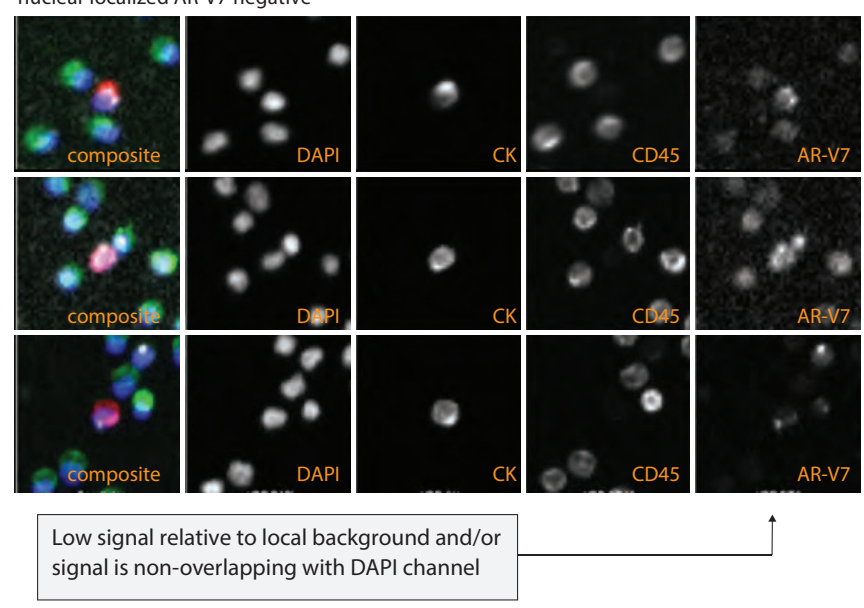

Fig. 3 - Scoring criteria for androgen receptor variant 7 (AR-V7) positive cells. Samples from 27 progressing mCRPC patients in need of a therapy change tested with the locked clinical trial assay prior to conduct of clinical utility studies. (A) Dot plot where every dot is one circulating tumor cell (CTC, blue) or CTC cluster (green); the y-axis indicates the immunofluorescence intensity and the x-axis indicates the unique sample identifier. Dashed line indicates the analytical cutoff for signal intensity, which combined with the presence of nuclearlocalized signal constitutes a positive. Representative AR-V7-positive (B) and AR-V7-negative (C) CTC images with the following panels left to right: 4-color composite, DAPI, CK, CD45, AR-V7. Exposure times are varied to maximize signal and fluorescence intensities are reported relative to the local background.

the locked assay to specifically detect the AR-V7 protein in CTCs from patients.

The three independent clinical studies performed after assay-lock showed the clinical utility of the Epic Sciences nuclear-localized AR-V7 assay in providing information for guiding treatment selection for men with progressing $\mathrm{mCRPCs}$ (9-11). Specifically samples were obtained at the pertinent decision point in management and collectively show that patients in whom nuclear-localized AR-V7 protein is identified in CTCs harbor tumors that are resistant to AATTs, such as abiraterone acetate or enzalutamide, and have a better chance at response and have longer survival times when treated with taxanes, such as docetaxel or cabazitaxel.

Other assays are currently available for AR-V7 detection of CTCs in the blood; however, only the nuclear-localized AR-V7 assay described here has met the Ballman criteria (19) for a predictive biomarker to date, in which two treatment groups, AATTs and taxanes, have been directly compared and a quantitative statistical interaction between biomarker, treatment group, and overall survival has been observed such as in the two studies reported by Scher et al $(9,10)$. The most recent study, PROPHECY $(11)$, a multicenter prospective trial, enrolled patients treated in the first and second lines with AATTs and directly compared AR-V7 protein expression by the nuclear-localized AR-V7 assay with the detection of AR-V7 mRNA using qPCR from isolated CTCs' affinity enriched from blood: the Johns Hopkins AR-V7 assay (18). The nuclear-localized protein expression AR-V7 assay identified fewer AR-V7-positive patients (11\% of patients having nuclear-localized AR-V7 protein vs. $24 \%$ having detectable AR-V7 mRNA in isolated (TC), although had a higher specificity of identifying nonresponse $(0 \%$ vs. $11 \% ; 50 \%$ PSA decline). While it is possible that some cells express AR-V7 mRNA, but not the functional proteins, both methods of AR-V7 detection showed a strong association with poor survival and measures of response, and 
increased in frequency at progression, providing further evidence that the AR-V7 splice variant is a mechanism of AATT resistance (11).

The AR-V7 protein is a transcription factor and is required to bind to DNA in order to activate the AR signaling axis, and with this in mind, prior tissue-based assessments of AR-V7 have generally required the criteria for nuclear localization (15-17). Similarly, for CTCs it is necessary that the AR-V7 protein is nuclear localized to make a positive biomarker call and in prior clinical outcome analysis it was found that the nuclear localization is required for making treatment decisions between AATTs and taxanes (13).

AATTs, such as abiraterone, enzalutamide, apalutamide, and darolutamide, have been shown to extend life in CRPC (1-7); however, these treatments are not curative and nearly all patients eventually progress (20). It is hypothesized that because the AR-V7 splice variant lacks the ligand binding domain it can track into the nucleus and activate the AR signaling axis in the absence of androgens. However, other mechanisms of resistance exist, including other forms of $A R$ reactivation, $A R$ bypass signaling, and $A R$ independent disease (20), and it is likely that AR-V7 expression may cooccur with many, highlighting the need for more comprehensive biomarkers that can be assessed in real time. Needed are additional blood-based CTC-based IF assays or genomic biomarkers assayed in CTCs or in circulating tumor DNA (ctDNA) to provide a more comprehensive biomarker panel to further inform therapy choice, where there are too few predictive biomarkers; the CTC nuclear-localized AR-V7 assay and tissue-based assessment of BRCA alterations for PARPi treatment are of only a few biomarkers to be reimbursed for physician choice in therapy selection to date.

\section{Disclosures}

Conflict of interest: D. Lu, R. Krupa, M. Harvey, R. Graf, J. Schonhoft, A. Jendrisak, A. Gill, S. Orr are or were employees of Epic Sciences during the writing, data collection, and analysis. H.I. Scher is a consultant/advisory board member for Ambry Genetics Corporation, Amgen, ESSA Pharma, Janssen Biotech, Janssen Research \& Development, OncLive Insights, Menarini Silicon Biosystems, Physicians Education Resource, Sanofi Aventis, and WCG Oncology; and he has received institutional research funding from Epic Sciences, Illumina, Janssen Diagnostics, Menarini Silicon Biosystems, and ThermoFisher. No other disclosures are reported.

Financial support: Epic Sciences provided facilities, materials, and services to perform isolation and sequencing of circulating tumor cells. Work at Memorial Sloan Kettering Cancer Center was supported by the Sidney Kimmel Center for Prostate and Urologic Cancers, and funded in part by the $\mathrm{NIH} / \mathrm{NCl}$ Cancer Center Support Grant to MSK (P30 CA008748), the NIH/NCI SPORE in Prostate Cancer grant to MSK (P50 CA092629), the Prostate Cancer Foundation, and the Prostate Cancer Clinical Trials Consortium.

\section{References}

1. Beer TM, Armstrong AJ, Rathkopf DE, et al. Enzalutamide in metastatic prostate cancer before chemotherapy. N Engl J Med. 2014;371:424-433.

2. Scher HI, Fizazi K, Saad F, et al. Increased survival with enzalutamide in prostate cancer after chemotherapy. N Engl J Med. 2012;367:1187-1197.
3. Ryan CJ, Smith MR, Fizazi K, et al. Abiraterone acetate plus prednisone versus placebo plus prednisone in chemotherapynaive men with metastatic castration-resistant prostate cancer (COU-AA-302): final overall survival analysis of a randomised, double-blind, placebo-controlled phase 3 study. Lancet Oncol. 2015;16:152-160.

4. de Bono JS, Logothetis CJ, Molina A, et al. Abiraterone and increased survival in metastatic prostate cancer. N Engl J Med. 2011;364:1995-2005.

5. Ryan CJ, Smith MR, de Bono JS, et al. Abiraterone in metastatic prostate cancer without previous chemotherapy. N Engl J Med. 2013;368:138-148.

6. Smith MR, Saad F, Chowdhury S, et al. Apalutamide treatment and metastasis-free survival in prostate cancer. N Engl J Med. 2018;378:1408-1418.

7. Fizazi K, Shore N, Tammela TL, et al. Darolutamide in nonmetastatic, castration-resistant prostate cancer. N Engl J Med. 2019;380:1235-1246.

8. George DJ, Sartor O, Miller K, et al. Treatment patterns and outcomes in patients with metastatic castration-resistant prostate cancer in a real-world clinical practice setting in the United States. Clin Genitourin Cancer. 2020;18:284-94.

9. Scher HI, Lu D, Schreiber NA, et al. Association of AR-V7 on circulating tumor cells as a treatment-specific biomarker with outcomes and survival in castration-resistant prostate cancer. JAMA Oncol. 2016;2:1441-1449.

10. Scher HI, Graf RP, Schreiber NA, et al. Assessment of the validity of nuclear-localized androgen receptor splice variant 7 in circulating tumor cells as a predictive biomarker for castrationresistant prostate cancer. JAMA Oncol. 2018;4:1179-1186.

11. Armstrong AJ, Halabi S, Luo J, et al. Prospective multicenter validation of androgen receptor splice variant 7 and hormone therapy resistance in high-risk castration-resistant prostate cancer: The PROPHECY Study. J Clin Oncol. 2019;37: 1120-1129.

12. Werner SL, Graf RP, Landers M, et al. Analytical validation and capabilities of the epic CTC platform: enrichment-free circulating tumour cell detection and characterization. J Circ Biomark. 2015;4:3.

13. Scher HI, Graf RP, Schreiber NA, et al. Nuclear-specific AR-V7 protein localization is necessary to guide treatment selection in metastatic castration-resistant prostate cancer. Eur Urol. 2017;71:874-882.

14. Hu R, Dunn TA, Wei $S$, et al. Ligand-independent androgen receptor variants derived from splicing of cryptic exons signify hormone-refractory prostate cancer. Cancer Res. 2009;69:16-22.

15. Efstathiou E, Titus M, Wen S, et al. Molecular characterization of enzalutamide-treated bone metastatic castration-resistant prostate cancer. Eur Urol. 2015;67:53-60.

16. Qu Y, Dai B, Ye D, et al. Constitutively active AR-V7 plays an essential role in the development and progression of castrationresistant prostate cancer. Sci Rep. 2015;5:7654.

17. Welti J, Rodrigues DN, Sharp A, et al. Analytical validation and clinical qualification of a new immunohistochemical assay for androgen receptor splice variant-7 protein expression in metastatic castration-resistant prostate cancer. Eur Urol. 2016; 70:599-608.

18. Antonarakis ES, Nakazawa M, Luo J. Resistance to androgen-pathway drugs in prostate cancer. N Engl J Med. 2014;371: 2234.

19. Ballman KV. Biomarker: predictive or prognostic? J Clin Oncol. 2015;33:3968-3971.

20. Watson PA, Arora VK, Sawyers CL. Emerging mechanisms of resistance to androgen receptor inhibitors in prostate cancer. Nat Rev Cancer. 2015;15:701-711. 